\title{
Novel Molecular Imaging of Atherosclerosis With Gallium-68-Labeled Apolipoprotein A-I Mimetic Peptide and Positron Emission Tomography
}

\author{
Emi Kawachi, PhD; Yoshinari Uehara, MD, PhD; Koki Hasegawa, PhD; Eiji Yahiro, MD, PhD; \\ Setsuko Ando, PhD; Yasuhiro Wada, BSc; Tsuneo Yano, PhD; \\ Hiroaki Nishikawa, MD, PhD; Masashi Shiomi, PhD; Shin-ichiro Miura, MD, PhD; \\ Yasuyoshi Watanabe, MD, PhD; Keijiro Saku, MD, PhD
}

\begin{abstract}
Background: High-density lipoprotein (HDL) plays a major role in reverse cholesterol transport. Many researchers have been working to enhance the biochemical function of HDL for use in therapy. Although HDL therapy using injections of apolipoprotein (apo)-A-I mimetics, apo A-I Milano or full-length apo A-I is dramatically effective, it is still unclear whether apo A-I or apo A-I mimetics actually enter atherosclerotic plaque and remove cholesterol from the lipid burden. We synthesized a novel 24-amino acid apo A-I mimetic peptide (known as FAMP) that potently removes cholesterol via specific ATP-binding cassette transporter A1. We then investigated the potential of FAMP to image developing plaque lesions in vivo.
\end{abstract}

Methods and Results: FAMP was modified with 1,4,7,10-tetraazacyclododecane-1,4,7,10-tetraacetic acid (DOTA) and radiolabeled with gallium-68 $\left({ }^{68} \mathrm{Ga}\right)$ for noninvasive positron emission tomography (PET) in an animal model (familial hypercholesterolemic myocardial infarction-prone rabbits: WHHL-MI) with atherosclerotic lesions. The ${ }^{68} \mathrm{Ga}-$ DOTA-FAMP was dramatically taken up by atherosclerotic tissues in the blood vessels and aorta of WHHL-MI rabbits, but not the control rabbits.

Conclusions: An apo A-I mimetic peptide with ${ }^{68} \mathrm{Ga}$-DOTA is a promising candidate diagnostic tracer for PET imaging of the atherosclerotic lipid burden and may contribute to the development of a tool for the diagnosis of plaque with PET. (Circ J 2013; 77: 1482-1489)

Key Words: Apo A-I mimetic peptide; Atherosclerosis; High-density lipoprotein; PET imaging; Plaque

A therosclerosis is a chronic, progressive local inflammatory disease that leads to vascular events, ${ }^{1}$ but most of the current anatomic imaging modalities can not show the active cellular and molecular processes that drive the evolution of plaque. ${ }^{2}$ Nuclear imaging is available through the use of positron emission tomography (PET), which is widely used for the imaging of atherosclerosis because of its superior sensitivity. Among the various approaches, fluoride-18 fluorodeoxyglucose ( $\left.{ }^{18} \mathrm{~F}-\mathrm{FDG}\right)$ is the most investigated tracer, but lacks molecular specificity for targeting atherosclerosis ${ }^{3,4}$ or aortitis. $^{5}$

\section{Editorial $\mathrm{p} 1403$}

High-density lipoprotein (HDL) plays a major role in reverse cholesterol transport (RCT) ${ }^{6,7}$ and clinical and epidemiological studies have demonstrated an inverse association between HDL cholesterol (HDL-C) and the risk of coronary events. Statin therapy increases plasma levels of HDL-C, ${ }^{6,8-10}$ and high-intensity statin therapy has been shown to reduce coronary plaque volume in patients with acute coronary syndrome, although this result was not predicted by any indices in the baseline lipid profile or the low-density lipoprotein cho-

Received June 5, 2012; revised manuscript received December 28, 2012; accepted January 20, 2013; released online March 2, 2013 Time for primary review: 29 days

Department of Cardiology, Fukuoka University School of Medicine, Fukuoka (E.K., Y.U., E.Y., H.N., S.M., K.S.); RIKEN Center for Molecular Imaging Science, Kobe (K.H., Y. Wada, T.Y., Y. Watanabe); Department of Community and Emergency Medicine, Fukuoka University, Fukuoka (E.Y.); Department of Chemistry, Faculty of Science, Fukuoka University, Fukuoka (S.A.); and Institute for Experimental Animals, Kobe University Graduate School of Medicine, Kobe (M.S.), Japan

The first four authors contributed equally to this work (E.K., Y.U., K.H., E.Y.).

Mailing address: Keijiro Saku, MD, PhD, FACP, FACC, FAHA, Department of Cardiology, Fukuoka University School of Medicine,

7-45-1 Nanakuma, Jonan-ku, Fukuoka 814-0180, Japan. E-mail: saku-k@fukuoka-u.ac.jp

ISSN-1346-9843 doi:10.1253/circj.CJ-12-0736

All rights are reserved to the Japanese Circulation Society. For permissions, please e-mail: cj@j-circ.or.jp 
lesterol (LDL-C) level at follow-up, but did correlate with the LDL-C/HDL-C ratio at follow-up. ${ }^{11}$ The RCT system has various potential antiatherogenic properties. For example, HDL particles transport cholesterol from the cells of the arterial wall, particularly from atherosclerotic plaque, to the liver. Many researchers are working to increase the quantitative levels of HDL-C and enhance the biochemical function of HDL as a therapeutic approach. Although injections of HDL apolipoprotein (apo) A-I mimetics, apo A-Imilano, or full-length apo A-I are effective both in vitro and in vivo, ${ }^{12,13}$ it is still unclear whether apo A-I or its mimetics actually enter atherosclerotic plaque and remove cholesterol. Such apo A-I may generate new HDL and reverse the macrophage-foam cell phenotype.

We synthesized a novel 24-amino acid apo A-I mimetic peptide without phospholipids, known as Fukuoka University apo A-I Mimetic Peptide [FAMP: inducible cholesterol efflux (iCE) peptide], which potently removes cholesterol in vitro via specific ATP-binding cassette transporter A1 (ABCA1). ${ }^{14}$ In that study using a cholesterol-fed mouse model, we found that FAMP promoted macrophage RCT. In the present study, we investigated the potential of FAMP for imaging plaque development in vivo.

\section{Methods}

\section{Synthesis of 1,4,7,10-Tetraazacyclododecane-1,4,7,10- Tetraacetic Acid (DOTA)-FAMP}

FAMP (FAMP type 5: H-ALEHLFTLYE KALKALEDLL KKLL-OH) and the scrambled-peptide of FAMP (Scramble: H-ATEFLHLLLLKLLKKKEDEL LAYA-OH) were modified using DOTA purchased from Strem Chemicals (Newburyport, MA, USA). DOTA-N-hydroxysuccinimidyl (NHS) ester was prepared by the preferential activation of 1 carboxyl group of the DOTA chelator in dimethyl sulfoxide (DMSO). ${ }^{15} \mathrm{~A}$ mixture of DOTA $(10 \mu \mathrm{mol})$, NHS $(10 \mu \mathrm{mol})$, and 1-ethyl-3-(3dimethylaminopropyl) carbodiimide hydrochloride (EDC: $10 \mu \mathrm{mol})$ in DMSO $(1 \mathrm{ml})$ was stirred at room temperature for $1 \mathrm{~h}$. A $50-\mu \mathrm{l}$ aliquot of this reaction mixture was then added to $50 \mu \mathrm{mol} / \mathrm{L}$ FAMP resin and stirred at room temperature for $1 \mathrm{~h}$. The resulting DOTA-FAMP resin was separated by reversedphase high-performance liquid chromatography and analyzed by matrix-assisted laser desorption/ionization time-of-flight mass spectrometry (MALDI-TOF MS; found: m/z 3,202.9 $(\mathrm{M}+\mathrm{H})+$, calculated for $(\mathrm{M}+\mathrm{H})+:$, 199.8). The predominant byproduct was confirmed to be an EDC-adduct derivative by MALDI-TOF measurement (Figure 1A).

DOTA-FAMP was confirmed to be stable when injected into rats, and the toxicity tests were also based on a standardized set of best practices and principles for conducting clinical laboratory studies (ie, GLP).

\section{Gallium-68 (68Ga)-Labeling of DOTA-FAMP}

DOTA-FAMP and DOTA-Scramble were radiolabeled with ${ }^{68} \mathrm{Ga}$ for use in noninvasive PET imaging. The ${ }^{68} \mathrm{Ga}$-DOTAFAMP was prepared using the method described by Zhernosekov et al. ${ }^{16}$ Briefly, a TiO 2 -based commercially available ${ }^{68} \mathrm{Ge} /{ }^{68} \mathrm{Ga}$ generator was eluted with $1 \mathrm{~N} \mathrm{HCl}$, and ${ }^{68} \mathrm{Ga}$ was purified using a cation exchange column (AG $50 \mathrm{~W}$-X8 cation exchange resin, 400- $\mu \mathrm{m}$ dry mesh; Bio-Rad Laboratories, Richmond, CA, USA). The ${ }^{68} \mathrm{Ga}$ was eluted with $3 \mathrm{ml} 98 \%$ acetone- $0.05 \mathrm{~mol} / \mathrm{L}$ $\mathrm{HCl}$ solution, after which the acetone in the eluent was removed under a nitrogen stream. DOTA-FAMP dissolved in $1 \mathrm{~mol} / \mathrm{L}$ of 4-(2-hydroxyethyl)-1-piperazineethanesulfonic acid (HEPES) buffer was added to the eluent, and the $\mathrm{pH}$ of this solution was adjusted to 3.5 by the addition of $1 \mathrm{~mol} / \mathrm{L}$
HEPES buffer. The reaction was performed for $15 \mathrm{~min}$ at $95^{\circ} \mathrm{C}$, and the reaction mixture was then passed over a Sep-Pak C18 column cartridge (Waters Corp, Milford, MA, USA), washed with $4 \mathrm{ml}$ water, and eluted with $400 \mu \mathrm{l}$ ethanol. Finally, the purified ${ }^{68}$ Ga-DOTA-FAMP and ${ }^{68}$ Ga-DOTA-Scramble solutions were evaporated under a nitrogen stream. ${ }^{68} \mathrm{Ga}$-DOTAFAMP and ${ }^{68} \mathrm{Ga}$-DOTA-Scramble were then dissolved in saline. The overall scheme for the preparation of ${ }^{68} \mathrm{Ga}$-DOTAFAMP is shown in Figure 1B.

\section{Animals}

We purchased 8 24-36-month-old Japanese white (JW) rabbits weighing 3.18-3.57 kg (F:M=2:6) from Japan SLC Inc (Shizuoka, Japan). In addition, 14 18-30-month-old myocardial infarction-prone homozygous Watanabe heritable hyperlipidemic (WHHL-MI) rabbits weighing 2.75-4.06 kg (F:M= 6:8; 2 were used for oil-red $\mathrm{O}$ staining) and 2 8-month-old WHHL-MI rabbits weighing 2.85-3.19kg $(\mathrm{F}: \mathrm{M}=1: 1)$ for the study of oil-red $\mathrm{O}$ staining with total cholesterol $>700 \mathrm{mg} / \mathrm{dl}$ were provided by Dr Shiomi, Kobe University, Japan. The rabbits were randomly divided into 2 groups: those to be injected intravenously with phosphate-buffered saline and those to be injected with ${ }^{68} \mathrm{Ga}-\mathrm{DOTA}-\mathrm{FAMP}$ (2-5 nmol per animal) and ${ }^{68} \mathrm{Ga}$-DOTA-Scramble (2-5 nmol per animal). The rabbits were maintained at Fukuoka University, Japan, and experiments were performed at the RIKEN Center for Molecular Imaging Science, Japan. The experiments complied with the regulations of the Committee on Ethics in the Care and Use of Laboratory Animals. The rabbits were housed in rooms maintained at $23 \pm 1^{\circ} \mathrm{C}$ and $55 \pm 5 \%$ relative humidity with free access to water and standard diet during acclimatization, and were fasted for $24 \mathrm{~h}$ before PET imaging.

\section{Stent Implantation in WHHL-MI Rabbits}

We used WHHL-MI rabbits for the vascular injury model, which was an atherosclerotic lesion in the left common iliac artery by stenting. The rabbits were anesthetized with $3 \%$ isoflurane, and a 4Fr sheath was then placed in the left carotid artery using the cut-down method. After cannulation, the stent $(2.5 \times 20 \mathrm{~mm}$; Boston Scientific Corp, Natick, MA, USA) was deployed in the left iliac artery at its nominal pressure (8-10 atm) for $30 \mathrm{~s}$ of balloon inflation under fluoroscopic guidance. In addition, heparin $(150 \mathrm{IU} / \mathrm{kg}$ ) was administered intravenously to the rabbits prior to catheterization. After stent implantation, angiography was performed to confirm the position of the stent. The skin incision was closed and the rabbits were allowed to recover from anesthesia. Four weeks later, the rabbits underwent PET imaging.

\section{Tracer Blood Clearance Study}

The pharmacokinetics of the tracer were determined in a JW and a WHHL-MI rabbit. Approximately $20 \mathrm{MBq}$ of ${ }^{68} \mathrm{Ga}-$ DOTA-FAMP was injected intravenously into one of the rabbit's ears, and blood $(0.2 \mathrm{ml})$ was drawn from the contralateral ear at 1, 3, 5, 10, 20, 40, 60, 90, 120, 180, 240, 300, and $360 \mathrm{~min}$. The radioactivity of the blood samples was measured using a Wallac 1480 Wizard 3', automatic gamma-counter (Perkin Elmer Japan Co Ltd, Kanagawa, Japan) and expressed as a percentage of the injected dose per gram.

\section{PET Imaging Protocol}

Imaging was carried out using a microPET ${ }^{\circledR}$ Focus220 (Siemens, Knoxville, TN, USA). The detector of this system is constructed from lutetium oxyorthosilicate and is $1.5 \times 1.5 \times 10 \mathrm{~mm}^{3}$. This system has 48 detector rings with a diameter of $26 \mathrm{~cm}$, an 


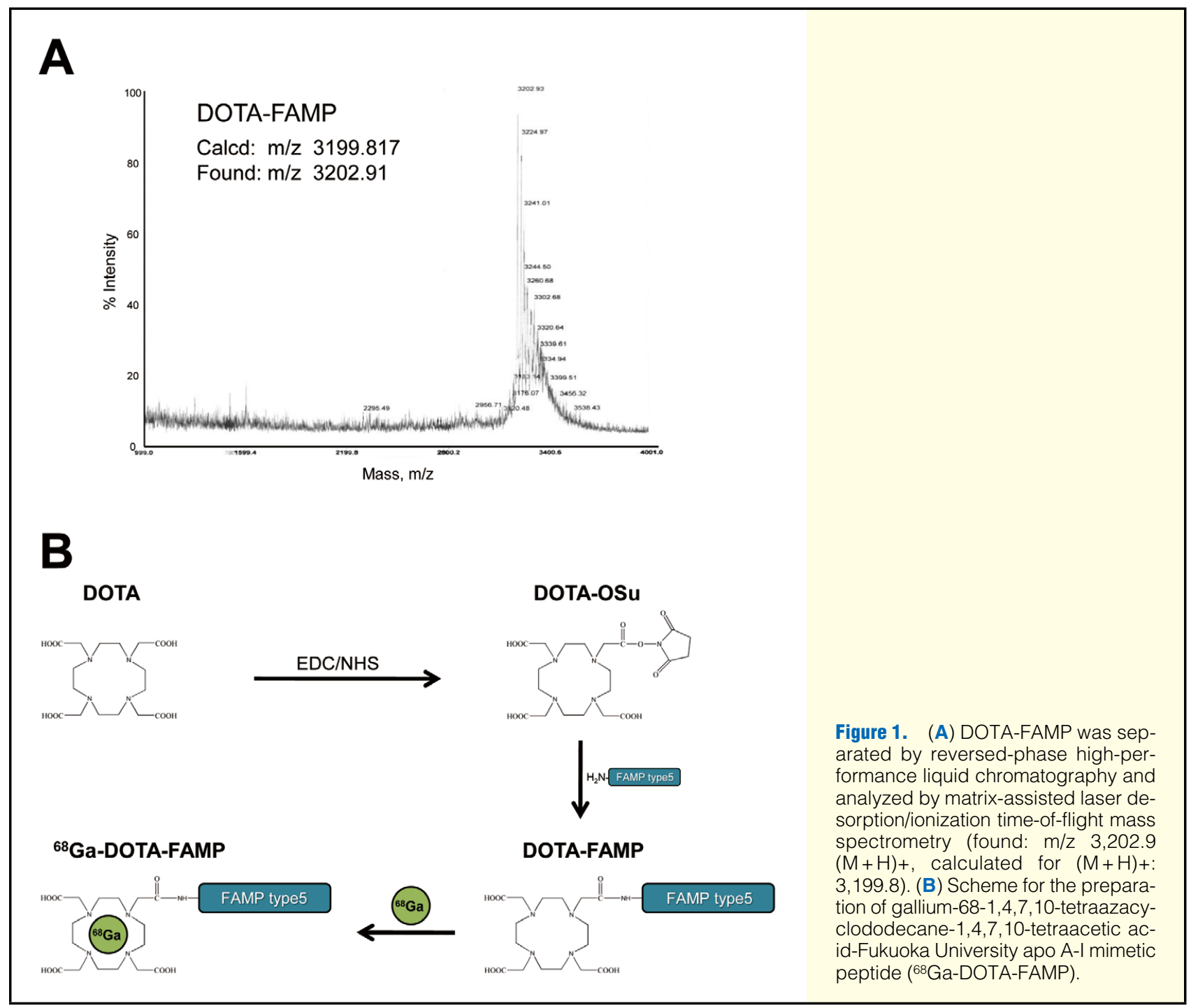

animal port with a diameter of $22 \mathrm{~cm}$, a capacity of 95 parallel slices, and a slice pitch of $0.80 \mathrm{~mm}$. Emission data acquisition was started at the same time as the intravenous administration of ${ }^{68} \mathrm{Ga}$-DOTA-FAMP for $360 \mathrm{~min}$ with an energy window of $400-650 \mathrm{keV}$ and a coincidence timing window of $6 \mathrm{~ns}$. The emission images were reconstructed by using filtered back projection with a Ramp filter and a cutoff at the Nyquist frequency. The time activity curves of the abdominal aorta (bifurcation of the aorta) region were obtained using ASIPro software included with the microPET system. ${ }^{68} \mathrm{Ga}$ activity in the aorta (bifurcation of the aorta) was continuously determined by PET for $6 \mathrm{~h}$ after the injection of ${ }^{68} \mathrm{Ga}$-labeled apo A-I mimetic peptide, and the results were adjusted for the background.

\section{Planar Positron Imaging System (PPIS) Analysis}

After the PET-CT scan, the rabbits were killed humanely and perfused with $1,000 \mathrm{ml} 0.9 \% \mathrm{NaCl}$. At $6 \mathrm{~h}$ after the injection ${ }^{68}$ Ga-DOTA-FAMP, extracted aortic tissues were scanned for 30 min using PPIS (Hamamatsu Photonics K.K., Shizuoka, Japan). Subsequently, the radioactivity of ascending and abdominal aorta sections from JW and WHHL-MI rabbits, respectively, was measured by gamma-counter.

\section{Evaluation of the Plaque and Radioactivity on Extracted Aorta}

${ }^{68} \mathrm{Ga}$-DOTA-FAMP was injected intravenously into WHHLMI rabbits [8 months old $(n=2)$, and 30 months old $(n=2)$ ] and $1 \mathrm{~h}$ later, the aorta was isolated and washed with saline 5 times. The extracted aortic tissues were scanned for $30 \mathrm{~min}$ using PPIS, and the same samples were subjected to oil-red $\mathrm{O}$ staining. Aortic tissues extracted from 4 different WHHL-MI rabbits were stained with oil-red $\mathrm{O}$. At $6 \mathrm{~h}$ after the injection of ${ }^{68} \mathrm{Ga}-\mathrm{DOTA}-\mathrm{FAMP}$, the radioactivity of plaque-rich or -poor lesions was measured by gamma-counter.

\section{Results}

The fractional catabolic rate (FCR) of plasma ${ }^{68} \mathrm{Ga}$-DOTAFAMP is shown in Figure 2A. The curve of the blood clearance of ${ }^{68} \mathrm{Ga}$-DOTA-FAMP for a JW rabbit showed that the radioactivity remaining in the blood at 1 and 5 min after injection was $0.262 \% \mathrm{ID} / \mathrm{g}$ and $0.319 \% \mathrm{ID} / \mathrm{g}$, respectively. This decreased sharply to $0.195 \% \mathrm{ID} / \mathrm{g}$ at $10 \mathrm{~min}$, and then decreased slowly to $0.122 \% \mathrm{ID} / \mathrm{g}$ and $0.079 \% \mathrm{ID} / \mathrm{g}$ at 120 and $360 \mathrm{~min}$ after injection, respectively. However, the curve for the WHHLMI rabbit showed residual activity in blood at 1 and $5 \mathrm{~min}$ 


\section{A}

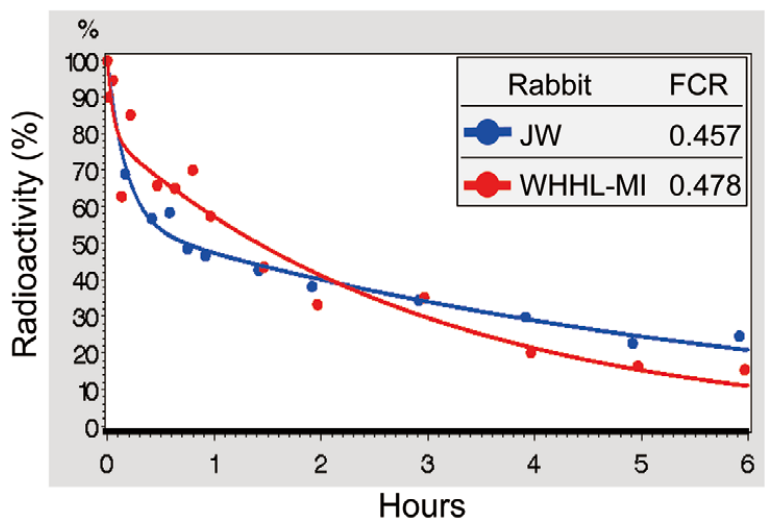

B

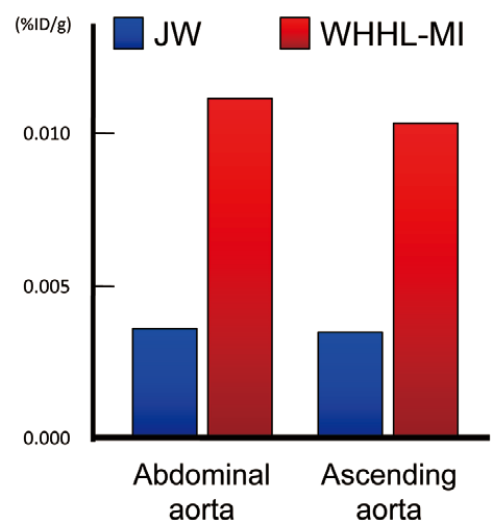

Figure 2. Blood clearance of ${ }^{68} \mathrm{Ga}$-DOTA-FAMP. (A) The fractional catabolic rate (FCR) of plasma ${ }^{8} \mathrm{G}$ G-DOTA-FAMP in Japanese white (JW) and myocardial infarction-prone Watanabe heritable hyperlipidemic (WHHL-MI) rabbits. (B) Radioactivity in the ascending and abdominal aortas extracted from JW and WHHL-MI rabbits at $6 \mathrm{~h}$ after the injection of ${ }^{68} \mathrm{Ga}-\mathrm{DOTA}$-FAMP. ${ }^{8} \mathrm{Ga}-\mathrm{DOTA}$, gallium-68-1,4,7,10-tetraazacyclododecane-1,4,7,10-tetraacetic acid; FAMP, Fukuoka University apo A-I mimetic peptide.

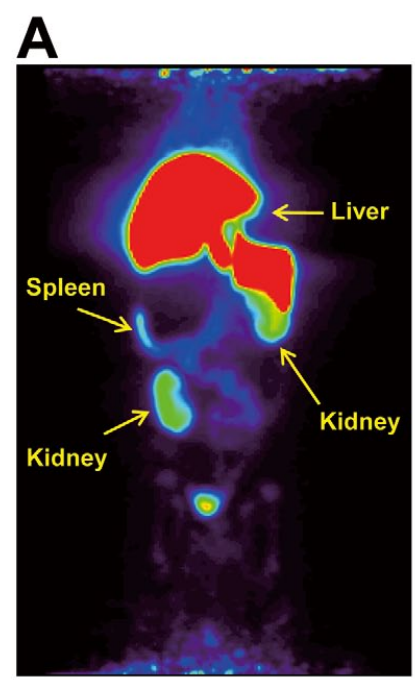

JW

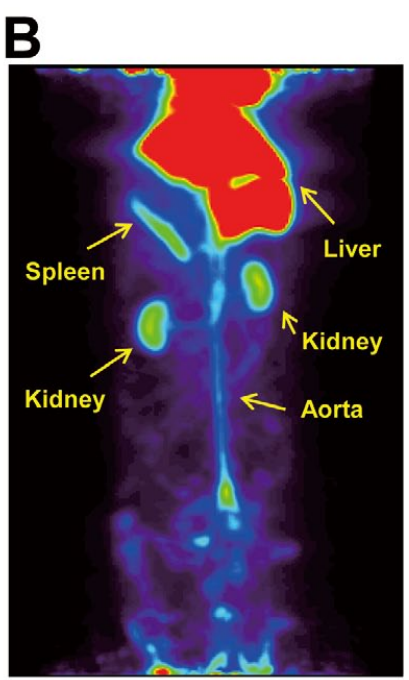

WHHL-MI

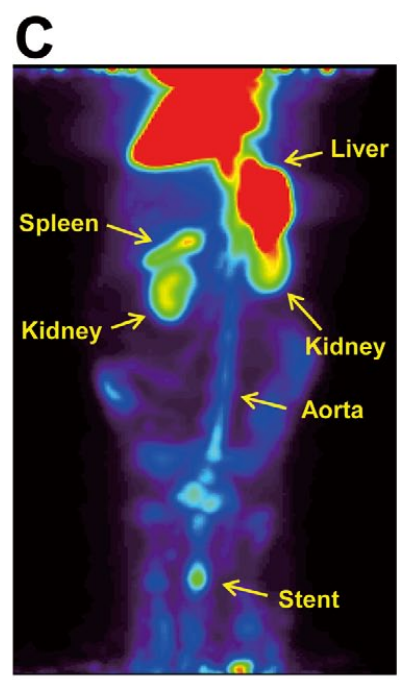

WHHL-MI

Figure 3. Noninvasive positron emission tomography (PET) imaging at $120 \mathrm{~min}$ after injection of the tracer reveals no uptake of ${ }^{68} \mathrm{Ga}$-DOTA-FAMP in the aorta of Japanese white (JW) rabbits (A), but there is aortic atherosclerotic plaque, represented by the high uptake of ${ }^{8} \mathrm{Ga}-\mathrm{DOTA}-\mathrm{FAMP}$, in the Watanabe heritable hyperlipidemic (WHHL-MI) rabbits (B). An extremely high-intensity signal of ${ }^{68} \mathrm{Ga}-\mathrm{DOTA}-\mathrm{FAMP}$ can be seen in the stenting lesion of the left common iliac artery in a WHHL-MI rabbit (C). ${ }^{88} \mathrm{Ga}-\mathrm{DOTA}$, gallium-68-1,4,7,10-tetraazacyclododecane-1,4,7,10-tetraacetic acid; FAMP, Fukuoka University apo A-I mimetic peptide.

after injection of $0.209 \% \mathrm{ID} / \mathrm{g}$ and $0.573 \% \mathrm{ID} / \mathrm{g}$, respectively. This decreased sharply to $0.381 \% \mathrm{ID} / \mathrm{g}$ at $10 \mathrm{~min}$, and then decreased slowly to $0.202 \% \mathrm{ID} / \mathrm{g}$ and $0.094 \% \mathrm{ID} / \mathrm{g}$ at 120 and $360 \mathrm{~min}$ after injection, respectively. Although the FCR of plasma ${ }^{68} \mathrm{Ga}$-DOTA-FAMP in WHHL-MI rabbits $(0.478)$ was slightly higher than that in JW rabbits $(0.457),{ }^{68} \mathrm{Ga}$-DOTAFAMP was drastically taken up by atherosclerotic tissue in the ascending and abdominal aorta of WHHL-MI rabbits (Figure 2B). At $120 \mathrm{~min}(2 \mathrm{~h})$ after the injection of ${ }^{68} \mathrm{Ga}-$ DOTA-FAMP, PET images clearly showed no uptake of the ${ }^{68} \mathrm{Ga}$-DOTA-FAMP tracer in the aorta of the JW rabbits (Figure 3A), but a high uptake in the aorta of WHHL-MI rabbits (Figure 3B). Furthermore, an extremely high uptake of ${ }^{68} \mathrm{Ga}$-DOTA-FAMP was observed in the stenting lesion of the 


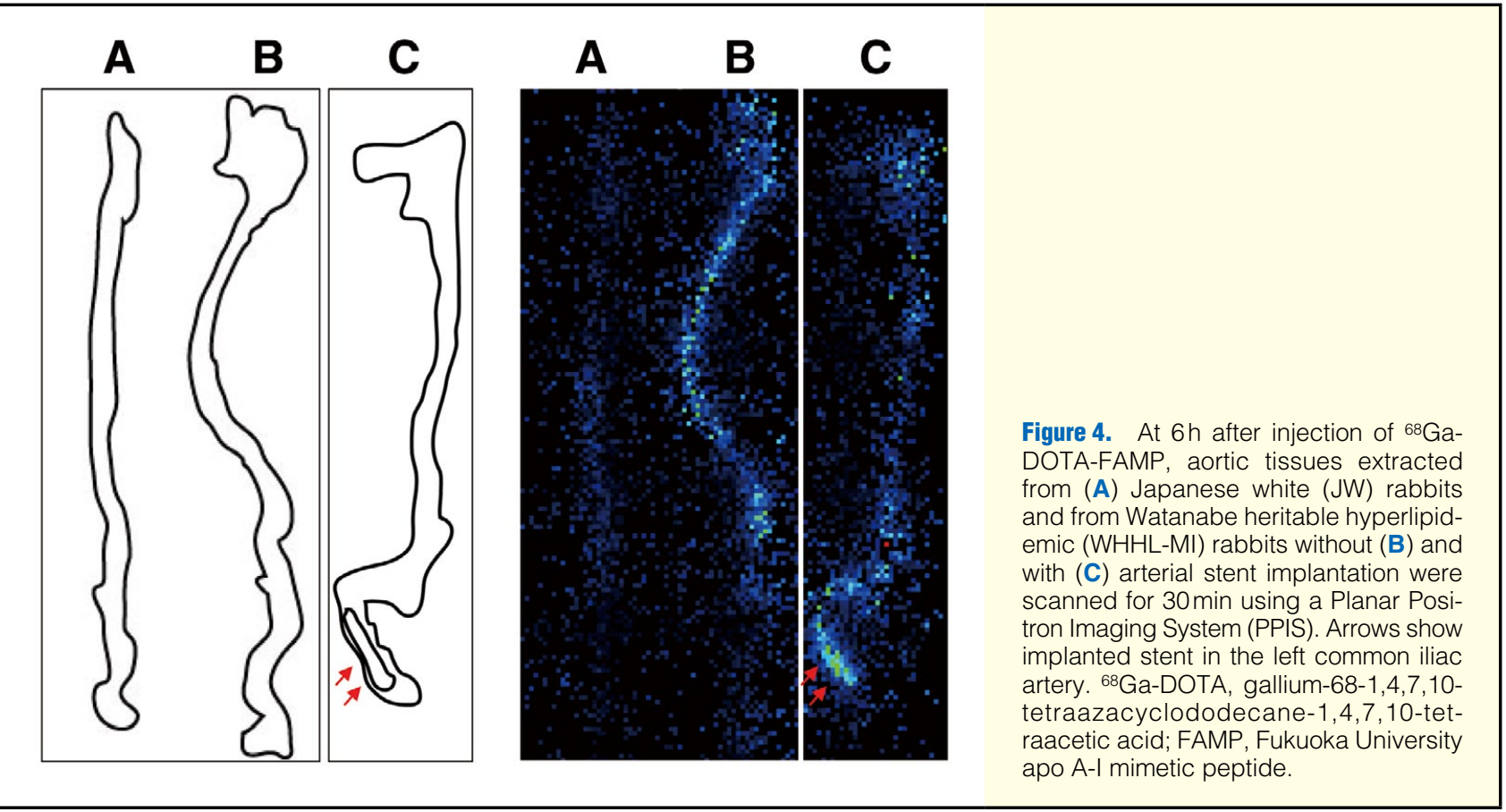

A

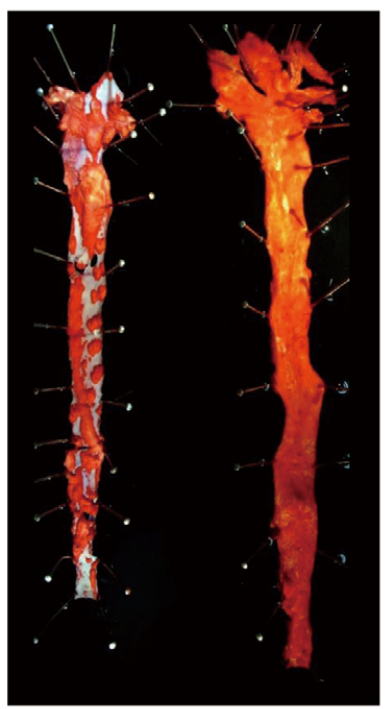

(a) (b)
B

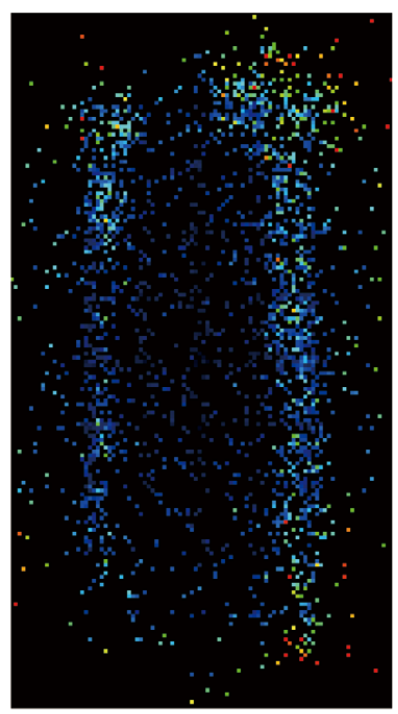

(a)

(b)
C

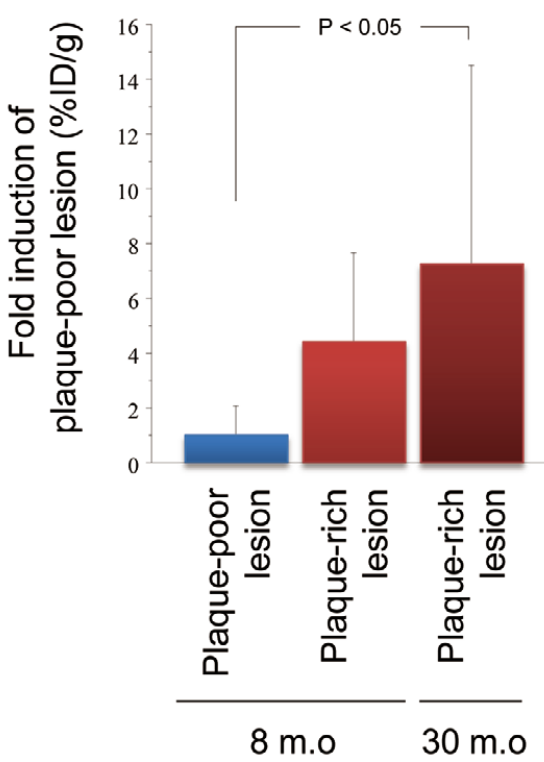

Figure 5. ${ }^{68} \mathrm{Ga}$-DOTA-FAMP was injected intravenously into myocardial infarction-prone homozygous Watanabe heritable hyperlipidemic (WHHL-MI) rabbits [8 months old (young, $n=2$ ), and 30 months old (old, $n=2)$ ]. At $1 \mathrm{~h}$ later, the aorta was isolated and washed with saline 5 times. The extracted aortic tissues were scanned for 30 min using a Planar Positron Imaging System (PPIS), and the same samples were subjected to oil-red $\mathrm{O}$ staining. (A) Aortic tissues extracted from 4 different WHHL-Ml rabbits stained with oil-red O. (B) PPIS analysis reveals high radioactivity in the extracted aorta of both the 8-month-old (8 m.o.) (a) and 30-monthold (30 m.o.) (b) rabbits. (C) At $6 \mathrm{~h}$ after injection of ${ }^{68} \mathrm{Ga}-\mathrm{DOTA}-\mathrm{FAMP}, 3$ samples of plaque-poor areas from each $8 \mathrm{~m} .0$. WHHL $(n=6), 5$ samples of plaque-rich areas from each 8 m.o. WHHL-MI $(n=10)$, and 7 samples of plaque-rich areas from each 30 m.o. WHHL-MI ( $n=14)$ rabbits were obtained. Radioactivity (\%ID)/g in a plaque-rich lesion (30 m.o. WHHL-MI) was significantly $(P<0.05)$ greater than in plaque-poor lesions (8 m.0. WHHL-MI). ${ }^{68} \mathrm{Ga}$-DOTA, gallium-68-1,4,7,10-tetraazacyclododecane-1,4,7,10-tetraacetic acid; FAMP, Fukuoka University apo A-I mimetic peptide. 
aortas of the WHHL-MI rabbits (Figure 3C). Consistent with the high uptake in the aorta of the WHHL-MI rabbits on PET imaging, PPIS analysis revealed that aortic sections extracted from these rabbits had high radioactive signals compared with those of JW rabbits (Figures 4A,B), and it also showed a high uptake of ${ }^{68} \mathrm{Ga}-D O T A-F A M P$ in the aortic stenting lesion in WHHL-MI rabbits (Figure 4C). Figure 5 shows the results of simultaneous oil-red $\mathrm{O}$ staining and PPIS imaging in young and old WHHL-MI rabbits. The plaque area showed a ${ }^{68} \mathrm{Ga}$ reaction. The result of oil-red $\mathrm{O}$ staining in the aorta of younger WHHL (Figure 5A-a) was similar to the PPIS result (Figure 5B-a), and lipid-poor lesions showed low PPIS activity. In old WHHL rabbits PPIS showed a stronger reaction (Figures 5A-b,B-b) compared with the younger WHHL rabbits (Figures 5A-a,B-a), although plaque and a strong radioactivity reaction were seen more often in the region of the aortic arch than in the lower descending aorta in both groups. The atherosclerosis in 30-month-old WHHL-MI rabbits progressed extensively and anatomically, but still trapped ${ }^{68} \mathrm{Ga}-$ DOTA-FAMP, whereas in the 8-month-old WHHL-MI rabbits there was mixed atherosclerosis with plaque-rich/-poor lesions. In addition to visualization by PPIS imaging, the radioactivity in oil-red $\mathrm{O}$ stained plaque lesions was greater than in plaque-poor tissues (Figure 5C). Moreover, tracer with the scrambled-peptide of FAMP did not show a signal by PET imaging in WHHL-MI rabbits (Figure 6). ${ }^{68}$ Ga-DOTA-Scramble was only taken up by the kidney, and not by the aorta or liver in WHHL-MI rabbits (Figure 6A), as compared with ${ }^{68} \mathrm{Ga}$-DOTA-FAMP (Figure 3B). The blood clearance of ${ }^{68} \mathrm{Ga}-$ DOTA-FAMP as a peptide tracer was relatively slow in both JW and WHHL-MI rabbits. We also performed a PET analysis $6 \mathrm{~h}$ after injection of ${ }^{68} \mathrm{Ga}$-DOTA-FAMP, when radioactivity would be completely cleared from the circulation. PET images of the abdominal aorta revealed a high accumulation of ${ }^{68} \mathrm{Ga}$-DOTA-FAMP in WHHL-MI rabbits compared with the control JW rabbits. As shown in Figure 7, the time-dependent attenuation of ${ }^{68} \mathrm{Ga}$-DOTA-FAMP in the abdominal aortic bifurcation in WHHL-MI rabbits (Figure 7B) was mild compared with that in JW rabbits (Figure 7A), whereas the scrambled-peptide disappeared immediately (Figure 7C). These results indicate that ${ }^{68} \mathrm{Ga}-\mathrm{DOTA}-\mathrm{FAMP}$ is taken up rapidly and remains in atherosclerotic tissue.

\section{Discussion}

We developed a novel PET tracer that was functionalized with DOTA and labeled with ${ }^{68} \mathrm{Ga}$ to specifically image the status of atherosclerotic plaque. In WHHL-MI rabbits, an atherosclerotic model of familial hypercholesterolemia, this novel tracer produced an impressive image of the aorta. Atherosclerotic plaque and aortic atherosclerotic plaque showed a high uptake of ${ }^{68} \mathrm{Ga}$-DOTA-FAMP in WHHL-MI rabbits (Figure 3B), but not JW rabbits (Figure 3A), by PET as well as PPIS methods (Figure 4). In addition to visualization by PPIS imaging, the radioactivity in oil-red $\mathrm{O}$ stained plaque lesions was greater than that in plaque-poor tissues (Figure 5C). The aortas extracted from old homologous WHHL rabbits were extremely sclerotic, such that we could not differentiate non-plaque from plaque lesions. However, we did observe plaque-poor lesions (Figure 5A-a) in younger WHHL rabbits. In WHHL-MI rabbits, a region that is visually determined to be non-plaque may in fact be a plaque-poor lesion, which is why the bifurcation of the aorta took up ${ }^{68}$ Ga-DOTA-FAMP. ${ }^{68}$ Ga-DOTA-FAMP is mainly taken up by the liver, and could re-enter the blood circulation after being absorbed by many organs; recycling of

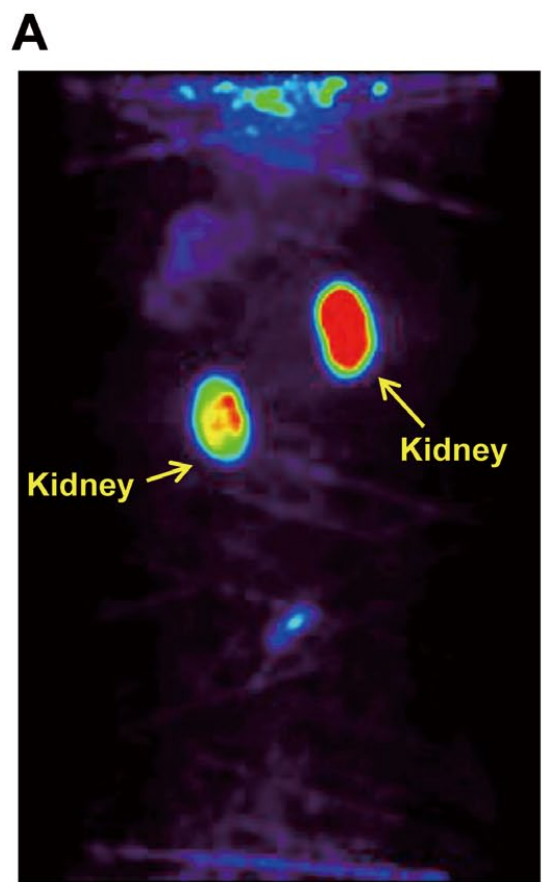

${ }^{68} \mathrm{Ga}-\mathrm{DOTA}-S c r a m b l e$

B

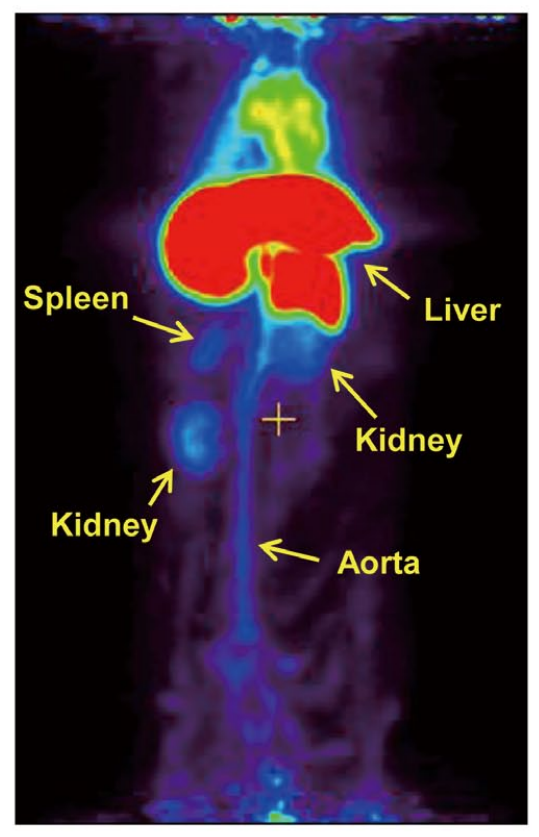

${ }^{68} \mathrm{Ga}-\mathrm{DOTA}-\mathrm{FAMP}$

Figure 6. Noninvasive positron emission tomography (PET) imaging with (A) ${ }^{68} \mathrm{Ga}$-DOTA-Scramble and (B) ${ }^{68} \mathrm{Ga}-\mathrm{DOTA}$ FAMP at $120 \mathrm{~min}$ after injection of the tracers in WHHL-MI rabbits shows high uptake of ${ }^{8} \mathrm{Ga}$-DOTA-FAMP in the liver, spleen and kidney, in addition to the aorta. In contrast, ${ }^{8} \mathrm{Ga}$ DOTA-Scramble shows high uptake in the kidney, but not the liver, spleen or aorta. ${ }^{68} \mathrm{Ga}$-DOTA, gallium-68-1,4,7,10-tetraazacyclododecane-1,4,7,10-tetraacetic acid; FAMP, Fukuoka University apo A-I mimetic peptide; Scramble, scrambledpeptide of FAMP; WHHL-MI, myocardial infarction-prone homozygous Watanabe heritable hyperlipidemic. 


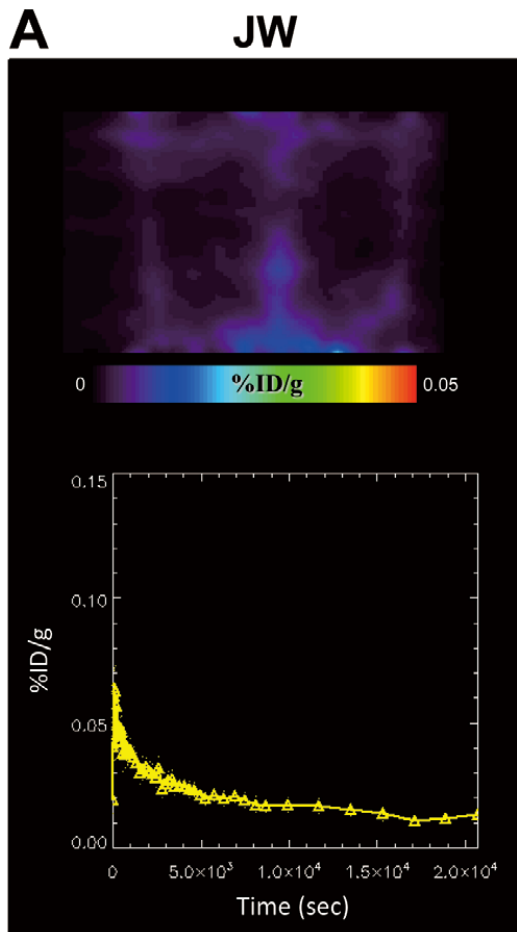

${ }^{68}$ Ga-DOTA-FAMP
B

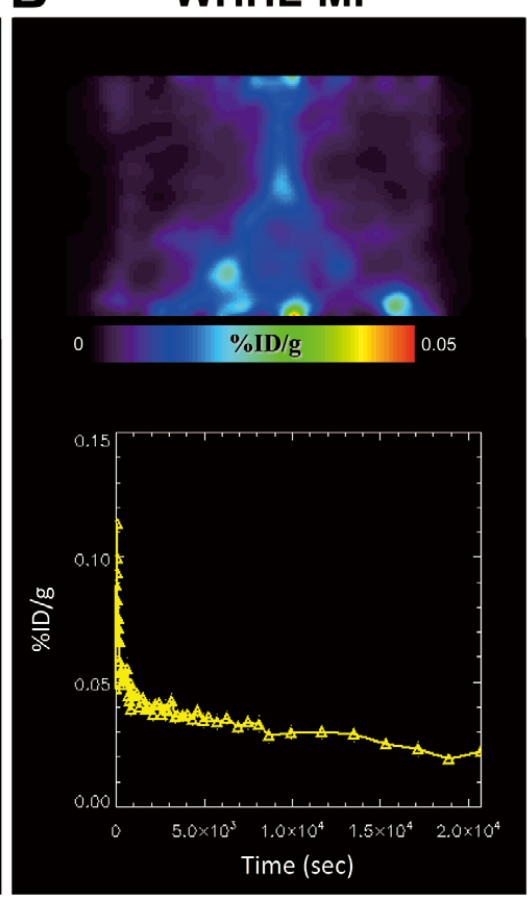

${ }^{68} \mathrm{Ga}-\mathrm{DOTA}$-FAMP

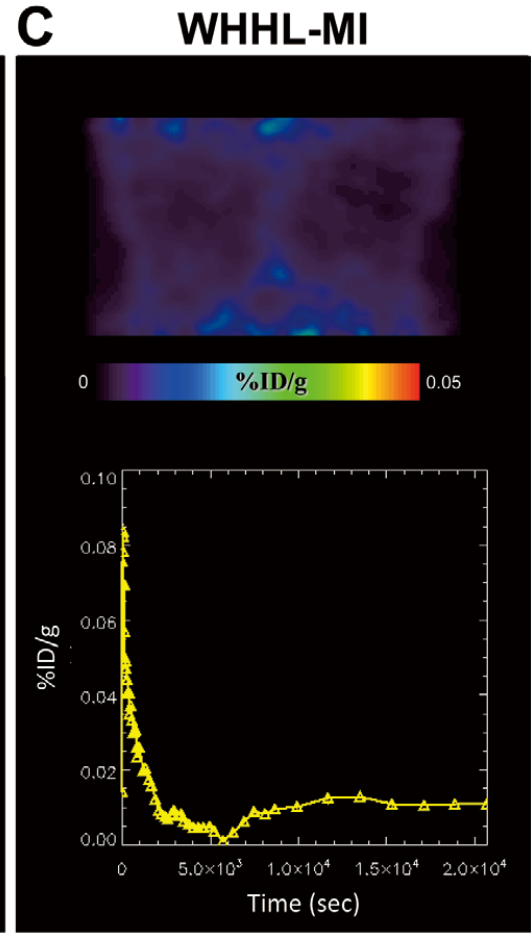

${ }^{68} \mathrm{Ga}-\mathrm{DOTA}$-Scramble

Figure 7. Time-dependent attenuation of ${ }^{68} \mathrm{Ga}$-DOTA-FAMP and ${ }^{8} \mathrm{Ga}$-DOTA-Scramble at the abdominal aortic bifurcation in (A) Japanese white (JW) and (B,C) myocardial infarction-prone homozygous Watanabe heritable hyperlipidemic (WHHL-MI) rabbits. Upper panels show continuous images of the abdominal aortic bifurcation for $6 \mathrm{~h}$ after the injection of tracers. ${ }^{68} \mathrm{Ga}$-DOTA, gallium68-1,4,7,10-tetraazacyclododecane-1,4,7,10-tetraacetic acid; FAMP, Fukuoka University apo A-I mimetic peptide; Scramble, scrambled-peptide of FAMP.

the tracer may be apparent under these conditions. Because the illumination of the aorta was more clear than with other imaging methods and ${ }^{68} \mathrm{Ga}-\mathrm{DOTA}-\mathrm{FAMP}$ interacts with plaque via a ABCA1 transporter, we consider that ${ }^{68}$ Ga-DOTA-FAMP provides HDL-functional imaging.

The PET images obtained using the ${ }^{68}$ Ga-DOTA-FAMP tracer show atherosclerotic plaques more clearly than those obtained using other reported tracers such as ${ }^{18} \mathrm{~F}-\mathrm{FDG}$ and ${ }^{111}$ In-low-density lipoprotein. ${ }^{17,18}$ Other candidate tracers, such as iodine-125-labeled monocyte chemoattractant peptide-1 or technetium-99 m-labeled anti-lectin-like oxidized LDL receptor-1 antibody, reportedly exhibit higher accumulation in atherosclerotic plaque. ${ }^{19,20}$ However, the amount of ${ }^{68} \mathrm{Ga}$-DOTAFAMP tracer injected in this study was far less than that of ${ }^{18} \mathrm{~F}-\mathrm{FDG}$ and other tracers in the previous studies. Our result shows that ${ }^{68} \mathrm{Ga}$-DOTA-FAMP may have greater specificity and sensitivity as a tracer, because the illumination of the aorta is sharper.

The target molecule for ${ }^{68} \mathrm{Ga}-D O T A-F A M P$ has not yet been identified, and may not be a specific molecule. The FAMP tracer removes cholesterol via the ABCA1 cholesterol transporter such as active apo A-I, and thereafter forms a pre- $\beta$ HDL particle. PET imaging demonstrated that the scrambledpeptide of FAMP was not taken up by the liver (Figure 6A), and its accumulation was immediately attenuated in the aortic bifurcation (Figure 7C) in contrast to FAMP. These observations suggest that FAMP has physiological characteristics similar to those of human apo A-I. Because iCE peptide FAMP has a specific function in removing cholesterol from cholesterol-rich lesions such as atherosclerotic plaque, ${ }^{68} \mathrm{Ga}-$ DOTA-FAMP might accumulate in target lesions requiring HDL or FAMP. Moreover, it is possible that ${ }^{68} \mathrm{Ga}-\mathrm{DOTA}-$ FAMP functions not only in mature, strongly inflamed atherosclerotic plaque, but also in the initial phase of atherosclerosis. Because PET imaging cannot include the heart, we targeted the descending aorta (bifurcation of aorta), which showed less atherosclerosis than we previously reported, ${ }^{21}$ and which was nicely viewed with PET (Figure 7B, Upper panel for WHHLMI). Conversely, both PET imaging and PPIS analysis of ${ }^{68} \mathrm{Ga}$-DOTA-FAMP showed strong radioactivity in the aortas extracted from WHHL-MI rabbits with atherosclerotic lesions. A high-intensity PPIS image was also positive in the newly stented vessel (Figure 4C), which showed that FAMP might bind to pro-inflammatory lesions with dysfunctional endothelium. Because the PPIS data showed high radioactivity in the aortic arch extracted from WHHL-MI, we could see changes in the uptake of ${ }^{68} \mathrm{Ga}$-DOTA-FAMP in the different stages of atherosclerosis (8- and 24-month-old WHHL rabbits). However, the region near the heart was not illuminated clearly, because the heart sequesters substantial radioactivity, which affects PET imaging. In addition to its role in promoting RCT, HDL has also been shown to have various pleiotropic effects ${ }^{22,23}$ such as antiinflammatory, ${ }^{20}$ antiapoptotic, ${ }^{24}$ antioxidant, ${ }^{25}$ and antithrombotic effects. ${ }^{26}$ Functional HDL and apo 
A-I have antiinflammatory properties. ${ }^{27-29}$ The present findings suggest that functional or pre- $\beta$ HDL plays a role in aortic plaque lesions with inflammatory changes.

\section{Study Limitations}

It is quite important to match PET-positive lesions with the results of immunohistochemical analysis to determine the cell make-up (CD31 for EC, Mac3 for macrophage foam cells etc). Detailed histochemical experiments could not be performed because of the difficulties associated with radioactive tissue.

\section{Conclusions}

We demonstrated that a unique apo A-I mimetic peptide with ${ }^{68} \mathrm{Ga}$-DOTA is a promising candidate diagnostic tracer for imaging the atherosclerotic lipid burden. Most researchers have focused on the therapeutic use of HDL, but the apo A-I mimetic peptide may contribute to the development of a tool for the diagnosis of plaque with PET.

\section{Acknowledgments}

This work was supported by grants-in-aid from the Ministry of Education, Science and Culture of Japan (No. 21590960, No. 24591123), the Fukuoka University One-Campus Project (2009, 2010, supported in part by the Ministry of Education, Science and Culture of Japan), a grant-inaid from the AIG Collaborative Research Institute of Cardiovascular Medicine, Fukuoka University, Japan, and ${ }^{\mathrm{NPO}}$ Clinical and Applied Science, Fukuoka, Japan.

\section{Disclosures}

None. K.S. is the Chief Director of NPO Clinical and Applied Science, Fukuoka, Japan.

\section{References}

1. Ross R. Atherosclerosis: An inflammatory disease. $N$ Engl J Med 1999; 340: 115-126.

2. Quillard T, Libby P. Molecular imaging of atherosclerosis for improving diagnostic and therapeutic development. Circ Res 2012; 111: $231-244$.

3. Rudd JH, Myers KS, Bansilal S, Machac J, Woodward M, Fuster V, et al. Relationships among regional arterial inflammation, calcification, risk factors, and biomarkers: A prospective fluorodeoxyglucose positron-emission tomography/computed tomography imaging study. Circ Cardiovasc Imaging 2009; 2: 107-115.

4. Rudd JH, Warburton EA, Fryer TD, Jones HA, Clark JC, Antoun N, et al. Imaging atherosclerotic plaque inflammation with $\left[{ }^{18} \mathrm{~F}\right]-$ fluorodeoxyglucose positron emission tomography. Circulation 2002; 105: $2708-2711$.

5. Ohigashi H, Haraguchi G, Konishi M, Tezuka D, Kamiishi T, Ishihara $\mathrm{T}$, et al. Improved prognosis of Takayasu arteritis over the past decade: Comprehensive analysis of 106 patients. Circ J 2012; 76: 1004 1011.

6. Gordon T, Castelli WP, Hjortland MC, Kannel WB, Dawber TR. High density lipoprotein as a protective factor against coronary heart disease: The Framingham study. Am J Med 1977; 62: 707-714.

7. von Eckardstein A, Nofer JR, Assmann G. High density lipoproteins and arteriosclerosis: Role of cholesterol efflux and reverse cholesterol transport. Arterioscler Thromb Vasc Biol 2001; 21: 13-27.

8. Assmann G. Genes and dyslipoproteinaemias. Eur Heart J 1990; 11(Suppl H): 4-8.

9. Briel M, Ferreira-Gonzalez I, You JJ, Karanicolas PJ, Akl EA, Wu P, et al. Association between change in high density lipoprotein cholesterol and cardiovascular disease morbidity and mortality: Systematic review and meta-regression analysis. BMJ 2009; 338: b92.

10. Saku K, Zhang B, Noda K, Investigators PT. Randomized head-to- head comparison of pitavastatin, atorvastatin, and rosuvastatin for safety and efficacy (quantity and quality of LDL): The PATROL trial. Circ J 2011; 75: 1493-1505.

11. Soeda T, Uemura S, Okayama S, Kawakami R, Sugawara Y, Nakagawa $\mathrm{H}$, et al. Intensive lipid-lowering therapy with rosuvastatin stabilizes lipid-rich coronary plaques: Evaluation using dualsource computed tomography. Circ J 2011; 75: 2621-2627.

12. Iwata A, Miura S, Zhang B, Imaizumi S, Uehara Y, Shiomi M, et al. Antiatherogenic effects of newly developed apolipoprotein A-I mimetic peptide/phospholipid complexes against aortic plaque burden in Watanabe-heritable hyperlipidemic rabbits. Atherosclerosis 2011; 218: $300-307$.

13. Nissen SE, Tsunoda T, Tuzcu EM, Schoenhagen P, Cooper CJ, Yasin $\mathrm{M}$, et al. Effect of recombinant apoA-I Milano on coronary atherosclerosis in patients with acute coronary syndromes: A randomized controlled trial. JAMA 2003; 290: 2292-2300.

14. Uehara $\mathrm{Y}$, Ando S, Oniki K, Abe S, Yahiro E, Tanigawa H, et al. FAMP, a powerful producer of pre-beta HDL via an ABCA1-mediated cholesterol efflux pathway. Circulation (Meeting Abstr Suppl) 2010; 122: A12493.

15. Kamei N, Morishita M, Kanayama Y, Hasegawa K, Nishimura M, Hayashinaka E, et al. Molecular imaging analysis of intestinal insulin absorption boosted by cell-penetrating peptides by using positron emission tomography. J Control Release 2010; 146: 16-22.

16. Zhernosekov KP, Filosofov DV, Baum RP, Aschoff P, Bihl H, Razbash AA, et al. Processing of generator-produced $68 \mathrm{Ga}$ for medical application. J Nucl Med 2007; 48: 1741-1748.

17. Rosen JM, Butler SP, Meinken GE, Wang TS, Ramakrishnan R, Srivastava SC, et al. Indium-111-labeled LDL: A potential agent for imaging atherosclerotic disease and lipoprotein biodistribution. $\mathrm{J} \mathrm{Nucl}$ Med 1990; 31: 343-350.

18. Worthley SG, Zhang ZY, Machac J, Helft G, Tang C, Liew GY, et al. In vivo non-invasive serial monitoring of FDG-PET progression and regression in a rabbit model of atherosclerosis. Int $J$ Cardiovasc Imaging 2009; 25: 251-257.

19. Ishino S, Mukai T, Kuge Y, Kume N, Ogawa M, Takai N, et al. Targeting of lectinlike oxidized low-density lipoprotein receptor 1 (LOX-1) with 99mTc-labeled anti-LOX-1 antibody: Potential agent for imaging of vulnerable plaque. J Nucl Med 2008; 49: 1677-1685.

20. Ohtsuki K, Hayase M, Akashi K, Kopiwoda S, Strauss HW. Detection of monocyte chemoattractant protein-1 receptor expression in experimental atherosclerotic lesions: An autoradiographic study. Circulation 2001; 104: 203-208.

21. Saku K, Fujino M, Yamamoto K, Ying H, Tashiro N, Harada R, et al. Cardiac function of WHHL rabbit, an animal model of familial hypercholesterolemia. Artery 1990; 17: 271 -280.

22. Eisenberg S. High density lipoprotein metabolism. J Lipid Res 1984; 25: $1017-1058$.

23. Linsel-Nitschke P, Tall AR. HDL as a target in the treatment of atherosclerotic cardiovascular disease. Nat Rev Drug Discov 2005; 4: $193-205$

24. Terasaka N, Wang N, Yvan-Charvet L, Tall AR. High-density lipoprotein protects macrophages from oxidized low-density lipoproteininduced apoptosis by promoting efflux of 7-ketocholesterol via ABCG1. Proc Natl Acad Sci USA 2007; 104: 15093-15098.

25. Goswami B, Tayal D, Gupta N, Mallika V. Paraoxonase: A multifaceted biomolecule. Clin Chim Acta 2009; 410: 1-12.

26. Mineo C, Deguchi H, Griffin JH, Shaul PW. Endothelial and antithrombotic actions of HDL. Circ Res 2006; 98: 1352-1364.

27. Navab M, Hama SY, Anantharamaiah GM, Hassan K, Hough GP, Watson AD, et al. Normal high density lipoprotein inhibits three steps in the formation of mildly oxidized low density lipoprotein: Steps 2 and 3. J Lipid Res 2000; 41: 1495-1508.

28. Rye KA, Bursill CA, Lambert G, Tabet F, Barter PJ. The metabolism and antiatherogenic properties of HDL. J Lipid Res 2009; 50(Suppl): S195-S200.

29. Navab M, Imes SS, Hama SY, Hough GP, Ross LA, Bork RW, et al. Monocyte transmigration induced by modification of low density lipoprotein in cocultures of human aortic wall cells is due to induction of monocyte chemotactic protein 1 synthesis and is abolished by high density lipoprotein. J Clin Invest 1991; 88: 2039-2046. 